\title{
Crosstalk Error Analysis in IIDFC Readout Circuit for Use in Piezoresistive Composite
}

\author{
Fabian Castro, Thiago Pentiado, Jorge Blanco, Ricardo Xavier, Marcelo Sanches, and Aparecido de Carvalho
}

\begin{abstract}
Resistive sensor arrays (RSAs) have been developed for a variety of applications depending on resolution requirements, active sensor area, and cost. Composite-based piezoresistive sensors are commonly found in RSAs. However, they present an intrinsic problem related to the parasitic current paths that flow through the whole composite, which cause a crosstalk effect. Various circuits have been proposed to reduce the crosstalk effect in sensor arrays. In this paper, we compare a number of readout circuits with PSpice models, mainly those including voltage feedback (VF) circuits and zero potential (ZP) circuits. To develop an array with 3600 sensors using a piezoresistive composite, we selected the improved isolated drive feedback circuit $(I I D F C)$. Using nodal analysis, we were able to develop an alternative mathematical circuit model, which allowed us to identify its unique characteristics, including the influence of array size on the crosstalk effect and the restriction on minimal impedance that the array elements must attain to maintain a minimum error. In a resistive array with 3600 sensors and a sensor range of $10-100 \mathrm{k} \Omega$, we obtained an absolute error of less than $3.56 \%$. In addition, the proposed mathematical circuit model obtained a maximum absolute error difference of $0.03 \%$ when compared with the PSpice model with the precision operational amplifier OPA37. Thus, using the IIDFC circuit and considering the minimal allowable impedance, it is possible to maintain a reduced error in an RSA with a large number of sensors.
\end{abstract}

Index Terms-Resistive sensor array, piezoresistive composite, crosstalk effect, minimal allowable impedance, improved isolated drive feedback circuit.

\section{INTRODUCTION}

A WIDE variety of sensors and applications have been developed by researches in an effort to improve health care in the population at large. In biomedical engineering, an important research direction involves viewing the foot as a fundamental human body part, since it is part of a kinematic chain that provides balance and stability while standing, walking, and running [1]-[3]. Research on the foot is conducted mainly through plantar pressure distribution analysis, using platforms that measure the ground contact forces over the sole of the foot, as well as using image processing techniques [4].

Today, the commercial equipment types used to measure the plantar pressure distribution are based on a variety of sensor technologies, including capacitive, resistive, piezoresistive and

Manuscript received August 22, 2017; revised October 17, 2017; accepted October 24, 2017. Date of publication November 8, 2017; date of current version December 7, 2017. This work was supported by Brazilian Councils CAPES and CNPq. The associate editor coordinating the review of this paper and approving it for publication was Dr. Pantelis Georgiou. (Corresponding author: Fabian Castro.)

The authors are with the Postgraduate Program in Electrical Engineering, UNESP-São Paulo State University, Ilha Solteira Campus, Ilha Solteira CEP 15385-000, Brazil (e-mail: fcastro12@gmail.com).

Digital Object Identifier 10.1109/JSEN.2017.2771151

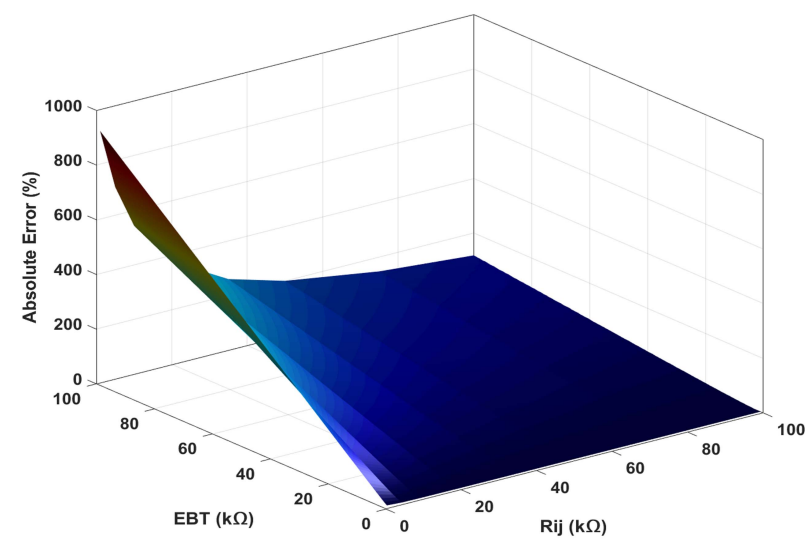

Fig. 1. Readout error while varying the EBT and the non-scanned elements of the array $\left(R_{i j}\right)$ in the range $1-100 \mathrm{k} \Omega$ with $\mathrm{M}=\mathrm{N}=4$.

piezoelectric technologies, among others [4]. However, this equipment is often expensive, which makes its acquisition beyond the reach of research groups and even health care institutions [5]. As such, low cost equipment has been developed that primarily uses resistive sensors [6]-[8]. However, this equipment has a low spatial resolution and its active area is limited to an area not much larger than the foot size.

One solution to the problem of low cost, resolution and size of the active area is the use of sensors based on piezoresistive composites, which are widely used in the robotics field and also to measure plantar pressure distributions [9]-[11].

Piezoresistive sensors consist of a conductive polymer located between two conductive layers, which are aligned perpendicularly to make a sensor array with $\mathrm{M} \times \mathrm{N}$ number of sensors, where $\mathrm{M}$ is the number of rows and $\mathrm{N}$ the number of columns. Therefore, it is possible to increase the sensor resolution by varying the size and quantity of the conductive rows and columns [12], [13].

Although the resistive sensor array (RSA) reduces the number of complex connections by sharing rows and columns, when a particular sensor must be read, the RSA exhibits a crosstalk effect,which is caused by parasitic current paths that flow through the non-scanned elements in the RSA [14], [15].

In this study, we implemented the circuit used by Castro [5], which has an RSA of $\mathrm{M}=\mathrm{N}=4$ and no diodes, in the PSpice environment. By scanning all elements of the array, including the element being tested $(E B T)$ and the non-scanned elements $\left(R_{i j}\right)$, we verified the crosstalk effect on the readout of the EBT. The array elements vary from $1 \mathrm{k} \Omega$ to $100 \mathrm{k} \Omega$. As shown in the Fig. 1, the greatest error (921\%) occurs when 
the EBT has a high impedance $(100 \mathrm{k} \Omega)$ and the non-scanned elements $R_{i j}$ have the lowest impedance ( $1 \mathrm{k} \Omega$ ).

As reported by Liu et al. [12] and Wu [16], to avoid or reduce the crosstalk effect, many circuits with different characteristics have been proposed over the years. Voltage feedback (VF) and zero potential (ZP) circuits are the most commonly used circuits in this respect.

In this work, we compare different readout circuitry technologies to identify the RSA circuit with the lowest percentage due to the crosstalk effect. Then, we select and mathematically model a circuit with the best response in terms of sensitivity, accuracy and implementation reproductivity, for projection in an RSA with $\mathrm{M}=\mathrm{N}=60$. In Section 2, we compare the readout circuits and in Section 3 we describe our mathematical model of the selected circuit. In Section 4, we present simulations and the characteristics of the selected circuit. Finally, we present a discussion and draw our conclusions in Section 5.

\section{CIRCUITS FOR SCANNING OF RESISTIVE SENSOR ARRAY}

As noted above in the previous section, there is a large error generated by the crosstalk effect in RSAs. To reduce this error, we must implement a readout circuit that is feasible, depending on the number and kind of elements required. Hence, we simulated in the PSpice environment a number of readout circuits referenced by $\mathrm{Wu}[16]$.

Circuit A, as proposed by D'Alessio [17], is a ZP circuit based on grounding, in which a buffer is connected to each column and there is one transresistance operational amplifier in each row. Thus, there is a zero potential difference on the non-scanned elements of the array (ZP-NSE), which decreases the crosstalk effect.

Circuit B, as proposed by Saxena et al. [18], is a ZP circuit with one operational amplifier (opamp) for all $\mathrm{MxN}$ resistive sensor array. In this case, the EBT is connected between the output and the inverting input of the opamp, and the noninverting input is grounded. The non-scanned elements of the selected column line ( $\mathrm{N}-1$ elements) are connected between the inverting input and the ground, and the non-scanned elements of the selected row line (M-1 elements) are connected between the output of the opamp and the ground. The remaining nonscanned elements $(\mathrm{M}-1) \times(\mathrm{N}-1)$ are grounded, i.e., with zero potential (ZP) difference. To set the circuit gain, we fixed one resistor $R_{L}$ between the input voltage and the inverting input of the opamp.

Circuit C, as proposed by Wu et al. [19], is a VF circuit with one opamp. In this circuit, the non-scanned elements of the non-selected rows and columns have the same potential difference, which is provided by the feedback voltage imposed for the system output voltage (VF-NSE). Conversely, the nonscanned elements of the selected row and column inject parasitic currents into the EBT, thereby increasing the crosstalk effect.

Similarly, Circuit D also proposed by Wu et al. [14] as an improvement upon Circuit $\mathrm{C}$, is based on a special feedback configuration that allows for a reduction in the error caused by the non-scanned elements on the selected column.

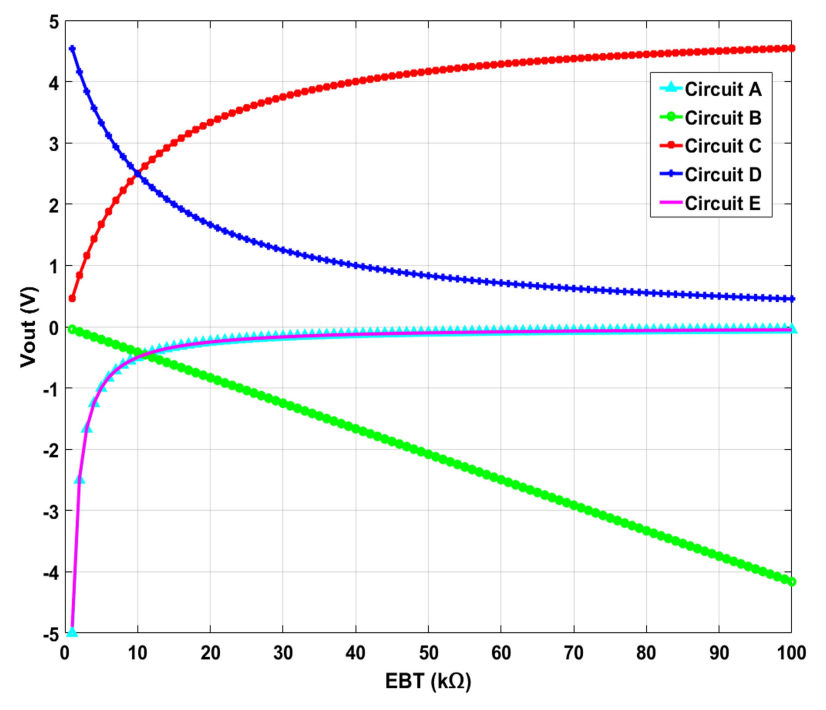

Fig. 2. Output voltages, varying EBT from $1-100 \mathrm{k} \Omega$ and $R_{i j}=100 \mathrm{k} \Omega$.

However, the adjacent row elements continue to influence the EBT readout. To reduce this behavior, Wu et al. [20] proposed an improvement for Circuit D, but this new circuit theoretically require two sampling channels to reduce the crosstalk effect, thereby increasing the RSA sampling time.

Finally, Circuit E, as proposed by $\mathrm{Wu}$ et al. [21], is a ZP circuit that enhances the setting non-scanned-electrode zero potential circuit (S-NSE-ZPC) proposed by Liu et al. [12]. In this case, two operational amplifiers are used to ensure the same ZP difference between all the non-scanned elements.

To perform an objective comparison, we simulated the abovementioned circuits under the following conditions: all voltage outputs were in $0-5 \mathrm{~V}$ range; input voltages $V_{i}=5 \mathrm{~V}$; conduction impedances of switches, multiplexers and demultiplexers were set to $R_{S W}=10 \Omega$ as suggested by Kim et al. [15]; we used general purpose operational amplifiers like the LM741 with a supply voltage $\pm 12 \mathrm{~V}$ and the impedances associated with each circuit were as follows Circuit A: $R_{r}=1 \mathrm{k} \Omega$; Circuit B: $R_{L}=120 \mathrm{k} \Omega$; Circuit C: $R_{S}=10 \mathrm{k} \Omega$; Circuit D: $R_{S}=10 \mathrm{k} \Omega$; and Circuit E: $R_{C G}=50 \Omega, R_{L}=1 \mathrm{k} \Omega$.

Fig. 2 shows all the circuit output voltages that sweep the EBT and maintain the non-scanned elements in the array at high impedance, $R_{i j}=100 \mathrm{k} \Omega$. The output voltages in Circuits $\mathrm{A}$ and $\mathrm{E}$ are very similar, with high sensitivity in the range of $1-5 \mathrm{k} \Omega$ at $0.9500 \mathrm{~V} / \mathrm{k} \Omega$ and low sensitivity in the $20-100 \mathrm{k} \Omega$ range at just $0.0023 \mathrm{~V} / \mathrm{k} \Omega$. As such, we neglect Circuits A and E, since the piezoresistive composite can have a wide operating range from $10 \Omega$ to $100 \mathrm{M} \Omega$ [11].

To determine the circuit efficiency with respect to reducing the crosstalk effect, we evaluated the absolute percentage error on the readout voltage as follows:

$$
e \%=\left|\frac{V_{i d}-V_{\text {out }}}{V_{\text {id }}}\right| * 100
$$

for which we considered the output voltage in ideal conditions $V_{i d}$ (in the absence of parasitic currents) and the output voltage obtained by PSpice environment $V_{\text {out }}$, as suggested by D'Alessio [17]. 


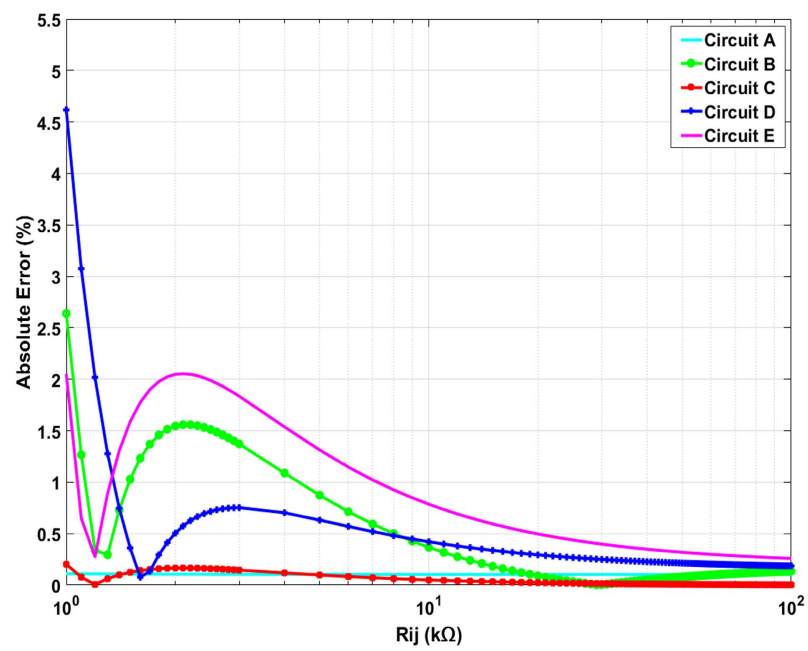

(a)

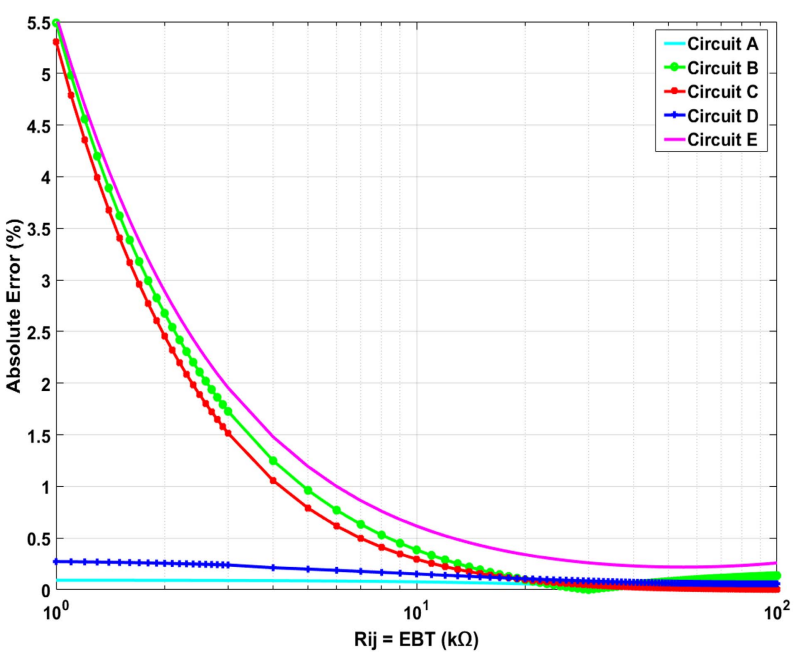

(b)

Fig. 3. Absolute percentage errors, (a) with $\mathrm{EBT}=100 \mathrm{k} \Omega$ and varying $R_{i j}$. (b) Varying simultaneously $\mathrm{EBT}=R_{i j}$. Variations of impedances are in range of $1-100 \mathrm{k} \Omega$.

Fig. 3 shows the absolute percentage error in the readout of the EBT. Fig. 3a shows the errors resulting when the nonscanned elements $R_{i j}$ vary and the EBT is fixed at high impedance. Fig. 3b show the errors when all array elements vary simultaneously $\left(E B T=R_{i j}\right)$. Although each circuit exhibits singular behavior, we can see that the lower is the value of the non-scanned elements of the array, the greater is the absolute error in the EBT readout.

By analysing the mean error of each circuit in Fig. 3a and Fig. $3 \mathrm{~b}$, it is possible to establish that Circuit $\mathrm{D}$ has the lowest mean error with $0.24 \%$, followed by Circuit $\mathrm{C}$ with $0.29 \%$ and finally the Circuit B with $0.50 \%$. Although the Circuit A has a mean error of $0.08 \%$, it was rejected because of its sensitivity.

Hence, we selected the Improved Isolated Drive Feedback Circuit (IIDFC) here called Circuit D for the analysis of its use in a resistive array with 3600 sensors (60x60).

First, we simulated Circuit D using the abovementioned parameters, by varying the impedances of the non-scanned elements from $1 \mathrm{k} \Omega$ to $100 \mathrm{k} \Omega$, and increasing the array size to $\mathrm{M}=\mathrm{N}=60$. Secondly, we used (2) to evaluate the model

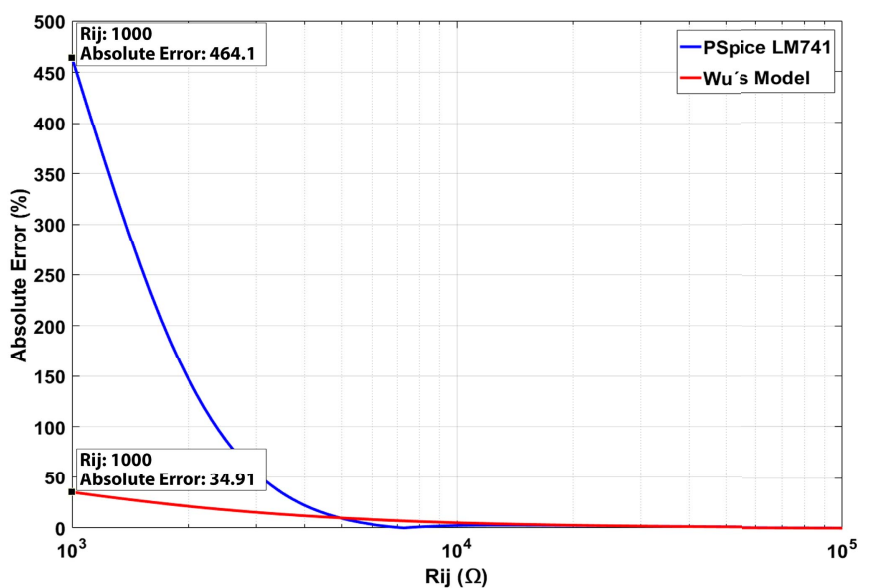

Fig. 4. Absolute errors, varying $R_{i j}$ from $1 \mathrm{k} \Omega$ to $100 \mathrm{k} \Omega$ and $\mathrm{EBT}=100 \mathrm{k} \Omega$, with $\mathrm{M}=\mathrm{N}=60$.

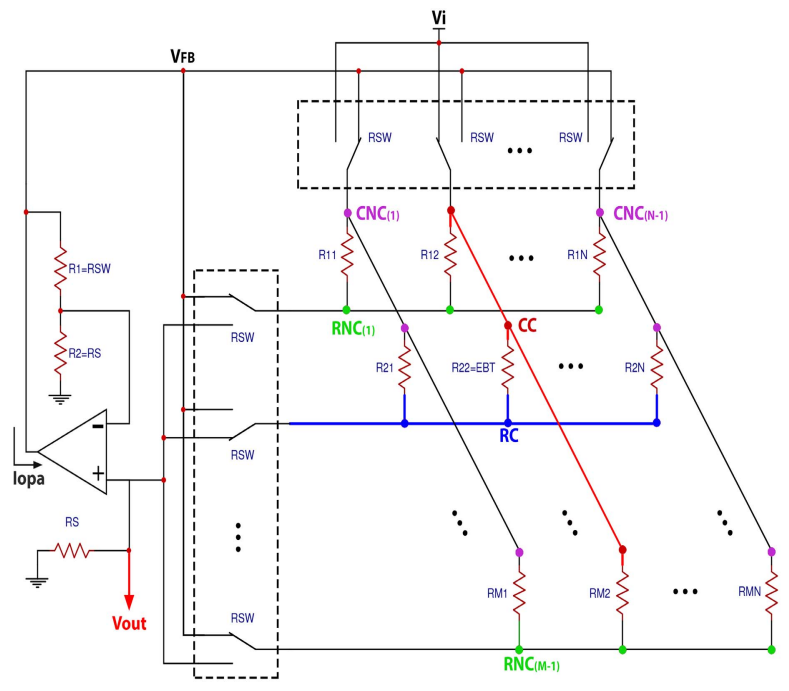

Fig. 5. Circuit D, improved isolated drive feedback circuit.

proposed by Wu et al. [14], considering that the non-scanned elements of the array have the same impedance $\left(R_{i j}\right)$ and all the conduction impedances of the switches, multiplexers and demultiplexers are also equal $\left(R_{S W}\right)$.

$$
V_{O U T}=\frac{R_{S} V_{i}}{R_{S}+2 R_{S W}+E B T\left(1+(M-1) \frac{R_{S W}}{R_{i j}}\right)}
$$

Fig. 4 shows the absolute errors obtained in the last two models(PSpice Model and Wu's Model), for which the maximal absolute error in PSpice model was $464.10 \%$, whereas Wu's model had an error of $34.91 \%$. We did not consider the output short circuit current in the operational amplifier in either case.

Due to the large difference between the theoretical and simulated models, to obtain a more accurate model that more closely resembles the simulated one, we must analyse in detail the electrical characteristics of Circuit D.

\section{Mathematical Analysis Circuit D (IIDFC)}

Fig. 5 shows Circuit D, as proposed by Wu et al. [14], which is an IIDFC. As described above, this circuit is a VF circuit in 
which the feedback voltage is the output voltage of the opamp, which is almost the same as the output voltage of the EBT. The feedback voltage is set on the rows and columns of the non-scanned elements of the array.

The model proposed by the authors in [14] via (2) does not consider the non-scanned elements on the column selected to read the EBT, and it also disregards all non-scanned elements in the non-selected rows and columns. Thus, by nodal analysis shown in Fig. 5, we obtained an alternative mathematical model to that proposed by the authors in [14], that allows us to evaluate unrelated characteristics of the circuit.

In Fig. 5, we can identify the main nodes as follows: the column-connected (CC) node, row-connected (RC) node, rownot-connected (RNC) node, and column-not-connected (CNC) node. With all non-scanned elements in the array having the same impedance $R_{i j}$, and since nodes on the non-selected columns have the same potential difference, as do the nodes on the non-selected rows, it is possible to obtain the system of linear equations expressed as (3):

$$
\begin{aligned}
V_{i}= & \left(1+\frac{R_{S W}}{E B T}+(M-1) \frac{R_{S W}}{R_{i j}}\right) V_{C C}-\left(\frac{R_{S W}}{E B T}\right) \\
& \times V_{R C}-\left((M-1) \frac{R_{S W}}{R_{i j}}\right) V_{R N C} \\
0= & V_{C C}-\left(1+\frac{E B T}{R_{S}+R_{S W}}+(N-1) \frac{E B T}{R_{i j}}\right) V_{R C} \\
& +\left((N-1) \frac{E B T}{R_{i j}}\right) V_{C N C} \\
0= & V_{C C}+\left(\frac{R_{i j}}{R_{S W}}\right) V_{R C}-\left(\frac{R_{i j}}{R_{S W}}+N\right) V_{R N C}+(N-1) V_{C N C} \\
0= & \left(1+\frac{R_{i j}}{R_{S W}}\right) V_{R C}+(M-1) V_{R N C}-\left(\frac{R_{i j}}{R_{S W}}+M\right) V_{C N C}
\end{aligned}
$$

We can use this system of linear equations in arrays of different sizes depending on the number of rows and columns ( $\mathrm{M}$ and $\mathrm{N}$ ). In this case, we used the ideal opamp model.

We can obtain the output voltage $V_{O U T}$ by solving (3). Then, we can define the output voltage as a function of the voltage at the RC node as follows.

$$
V_{O U T}=V_{R C} \frac{R_{S}}{R_{S}+R_{S W}}
$$

Since the system of linear equations mentioned above was solved using Matlab software, in this work, we refer to this solution as the Matlab model.

To obtain the absolute percentage error described in (1), we considered the ideal output voltage, since the ideal output is not influenced by the non-scanned elements in the array, and which we express as (5). We also added the conduction impedances of the switches, multiplexers and demultiplexers $\left(R_{S W}\right)$.

$$
V_{i d}=V_{i} \frac{R_{S}}{R_{S}+2 R_{S W}+E B T}
$$

In addition, the current provided by the opamp to the circuit is an important parameter that must be supervised, since excess current can cause saturation. The current provided by the opamp is defined as follows:

$$
\begin{array}{r}
I_{O P A}=\frac{-V_{F B}}{R_{S}+R_{S W}}+\frac{\left(V_{C N C}-V_{F B}\right)(N-1)}{R_{S W}} \\
+\frac{\left(V_{R N C}-V_{F B}\right)(M-1)}{R_{S W}}
\end{array}
$$

where $V_{F B}$ is the feedback voltage, which is described as follows.

$$
V_{F B}=V_{O U T}\left(1+\frac{R_{S W}}{R_{S}}\right)
$$

\section{VERIFICATION OF THE CIRCUIT D (IIDFC)}

\section{A. $4 \times 4$ Array}

To evaluate the decrease in the crosstalk effect error, we used a resistive array with $\mathrm{M}=\mathrm{N}=4$ and an IIDFC with the following parameters: non-scanned elements $R_{i j}$ ranging from $1 \mathrm{k} \Omega$ to $100 \mathrm{k} \Omega ; V_{i}=5.13 \mathrm{~V}$; conduction impedances of the switches, multiplexers and demultiplexers $R_{S W}=10.34 \Omega ; R_{S}=9.96 \mathrm{k} \Omega$ and $E B T=100.32 \mathrm{k} \Omega$, all these values were obtained with the Keysight 34405A 5 1/2 Digit Multimeter. We implemented the circuit using the general purpose operational amplifier LM741 and supply voltage of $\pm 12 \mathrm{~V}$. We also simulated two circuits with the same parameters in the PSpice environment, using the opamp LM741 and the precision operational amplifier OPA37.

The absolute errors of the these systems are shown in Fig. 6a, as well as the errors of the model proposed in (3) and the model proposed by Wu et al. [14] in (2). We emphasize that we obtained all errors as a function of the ideal output voltage established in (5).

The response of the Circuit D -IIDFC- in the PSpice environment with the opamp OPA37 had a mean error of $0.14 \%$ whereas the mean error of that with the opamp LM741 was $0.27 \%$, due to greater open loop gain of OPA37, which reduces the crosstalk effect, as suggested by Saxena et al. [18]. Similarly, the calculated mean error of the Matlab model is $0.11 \%$ and is very close to the response obtained in the PSpice environment with the opamp OPA37, with an error difference of only $0.03 \%$ between them. Conversely, the implemented circuit had a mean error of $1.31 \%$, which is the greatest error of all the model responses. However, this error was similar in its behavior to that of the other models, since the difference in its mean error and that of the PSpice environment and the Matlab model are $0.63 \%$ and $0.72 \%$ respectively.

Although the model proposed by Wu et al. [14] exhibited a mean error of only $0.13 \%$, it responded differently than the other models, which each had a minimal impedance where the absolute error becomes zero. Impedances lower than this minimal impedance cause a sudden increase in the error due to the crosstalk effect. This denotes the need for a restriction on the minimal value of the array elements to minimize any crosstalk effect, as also described by Saxena et al. [18].

In this way, by using the same parameters described above and by sweeping the impedances of the non-scanned elements of the array $\left(R_{i j}\right)$ in the $1 \mathrm{k} \Omega$ to $2 \mathrm{k} \Omega$ range, we can obtain a 


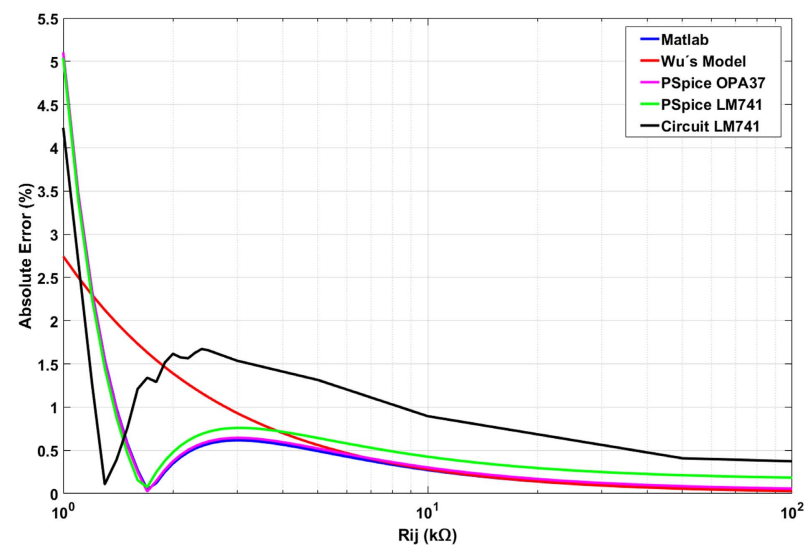

(a)

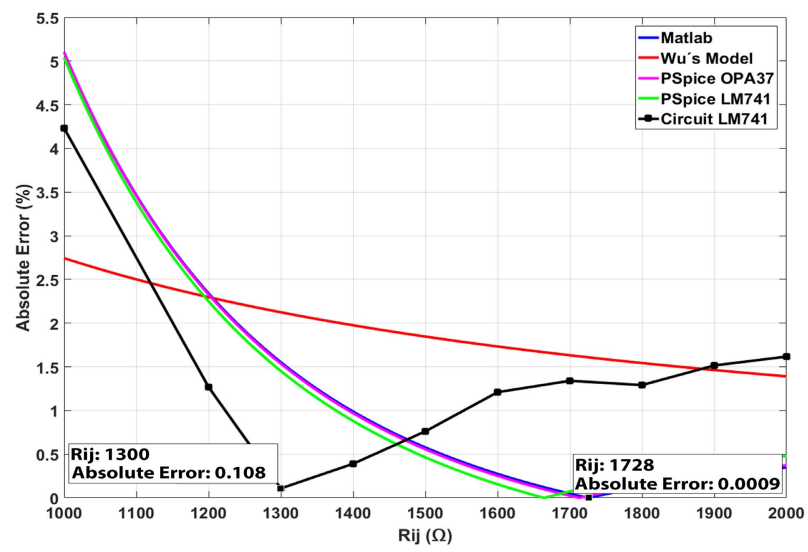

(b)

Fig. 6. (a) Absolute error of readout of EBT and varying $R_{i j}$. (b) Minimal impedance with $\mathrm{M}=\mathrm{N}=4$. $\mathrm{EBT}=100.32 \mathrm{k} \Omega$.

minimal impedance before experiencing any increase in the absolute error, as shown in Fig. 6b. Theoretically, we can determine the minimal impedance by equating (4) and (5).

According to data from the Matlab and PSpice models, with both the OPA37 and the LM741 opamps, the minimal impedance that ensures minimal errors is close to $1700 \Omega$, whereas the minimal impedance in the implemented circuit was close to $1300 \Omega$. This difference can be explained by the electrical characteristics of the LM741, the resistors used, and even the measuring instrument. Nonetheless, the implemented circuit obtained a lower minimal impedance than the other models, which is an advantage because it allows for a greater range of impedance in the RSA.

\section{B. $M \times N$ Array}

As described by $\mathrm{Wu}$ et al. [14], the array size has an influence on the readout of the EBT, i.e., the larger the array size, the greater the error caused by the crosstalk effect. Therefore, the number of rows in the array has a direct and significant influence on the readout error, as shown in Fig. 7a.

As reported by the authors in [14], the Circuit D -IIDFCreduces the influence of adjacent columns, that is, increasing the number of columns in the array has little influence on the readout error.

Likewise, since the array size influences the readout error of the EBT, it also influences the minimal impedance allowed by

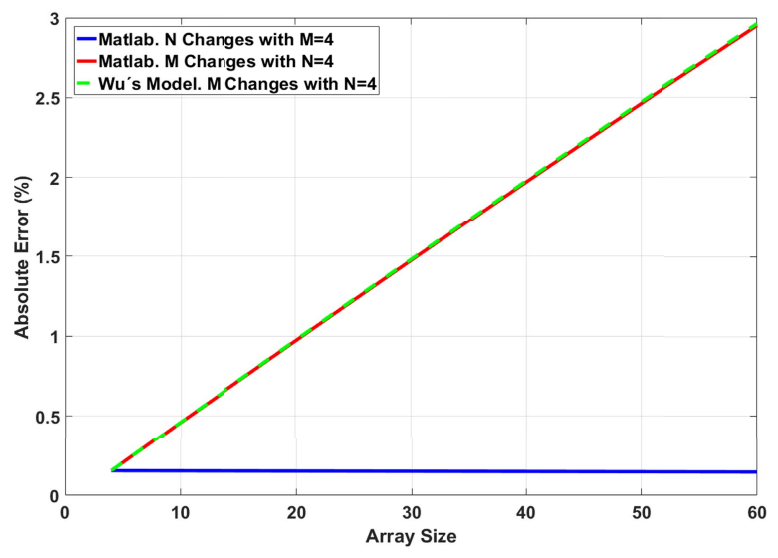

(a)

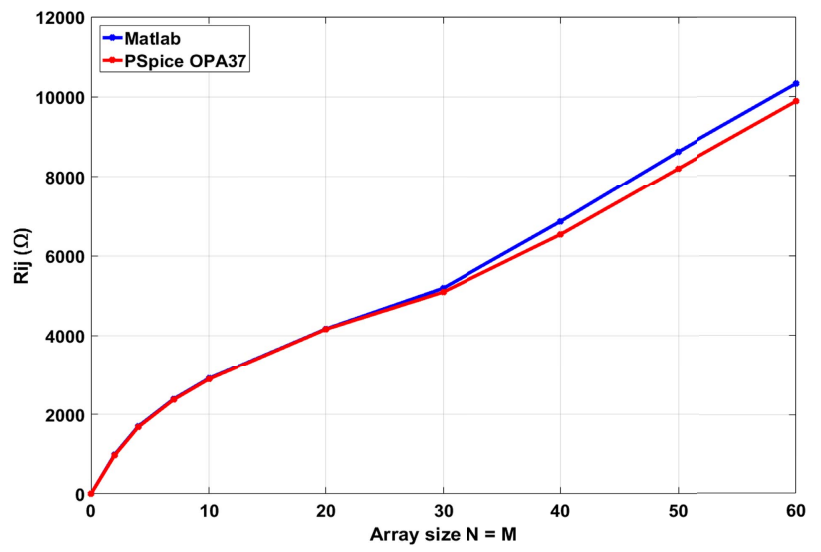

(b)

Fig. 7. (a) Array size effect on the readout error of the EBT. $R_{i j}=\mathrm{EBT}=10 \mathrm{k} \Omega$. (b) Minimal impedance as a function of array size with $\mathrm{N}=\mathrm{M}$ and $\mathrm{EBT}=100.32 \mathrm{k} \Omega$.

the circuit to avoid a considerable increment in the absolute error. The larger is the array size, the greater the allowable minimal impedance, as shown in Fig. 7b, via the Matlab and PSpice models.

As stated earlier, it is possible to determine the minimal impedance by equating (4) and (5). However, this is true only when the short circuit current of the opamp has not been reached. The maximal output drive supported by opamps OPA37 and LM741 is $25 \mathrm{~mA}$. So, by using the established parameters for the implemented circuit and sweeping the nonscanned elements $R_{i j}$ in (3), as well as supervising the current supplied by the opamp with (6), we can obtain the minimal allowable impedance to ensure low error without the opamp entering saturation mode. As shown in Fig. 7b, the opamp enters saturation mode with an approximately array size of $\mathrm{M}=\mathrm{N}=30$, thereby changing the natural circuit response and sharply increasing the minimal allowable impedance. It is important to note that the lower is the $R_{S W}$ value, the smaller the array size will be before the opamp enters saturation mode and the greater will be the minimal allowable impedance.

\section{Simulation Comparing Matlab and PSpice Models}

For verifying the IIDFC model proposed using (3), we compared it with the PSpice model using the opamp OPA37. 


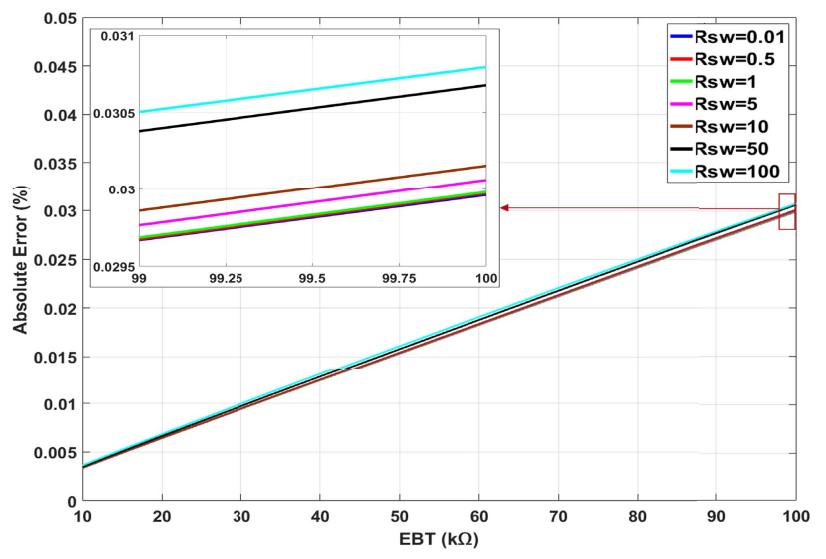

(a)

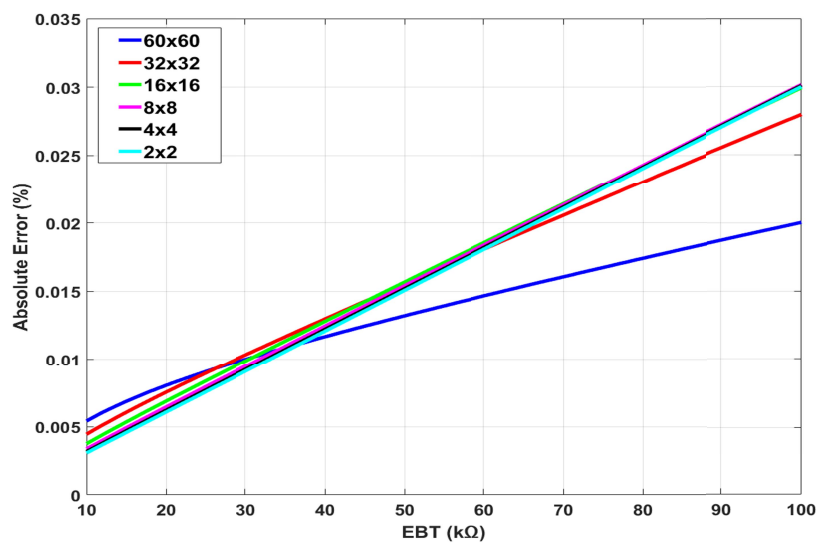

(b)

Fig. 8. (a) $R_{S W}$ effect on the difference between Matlab and PSpice models, where $\mathrm{N}=\mathrm{M}=8$. (b) Array size effect on the difference between Matlab and PSpice models, where $R_{S W}=10 \Omega$. Rij $=10 \mathrm{k} \Omega$ and EBT is in range of $10-100 \mathrm{k} \Omega$.

By varying the impedances in switches, multiplexers and demultiplexers $\left(R_{S W}\right)$ and the array size $(\mathrm{M}=\mathrm{N})$, we evaluated the percentage error between the models using (1), where the Matlab model is the theoretical value.

First, we evaluated the difference between the models by varying $R_{S W}$ in range of 0.01-100 $\Omega$. The non-scanned elements of the array $\left(R_{i j}\right)$ were fixed at $10 \mathrm{k} \Omega, \mathrm{M}=\mathrm{N}=8$, and the EBT varying from $10 \mathrm{k} \Omega$ to $100 \mathrm{k} \Omega$. The results are shown in Fig. 8a, where it is possible to note that the greater is the $R_{S W}$ value, the greater is the difference between the models. However, the percentage error between the models is lesser than $0.035 \%$ that is low enough to be consider.

Secondly, we evaluated the effect of the array size on the difference between the Matlab and PSpice models, by varying the array size with $\mathrm{M}=\mathrm{N}$, where $R_{S W}=10 \Omega, R_{i j}=$ $10 \mathrm{k} \Omega$ and the EBT is in range of $10-100 \mathrm{k} \Omega$. Once again, the error between the models is lesser than $0.030 \%$, as shown in Fig. 8b. Thus, the proposed model can be used for evaluating the IIDFC behavior.

\section{Piezoresistive Composite}

As described previously, piezoresistive composites facilitate the development of sensors with low cost, high resolution

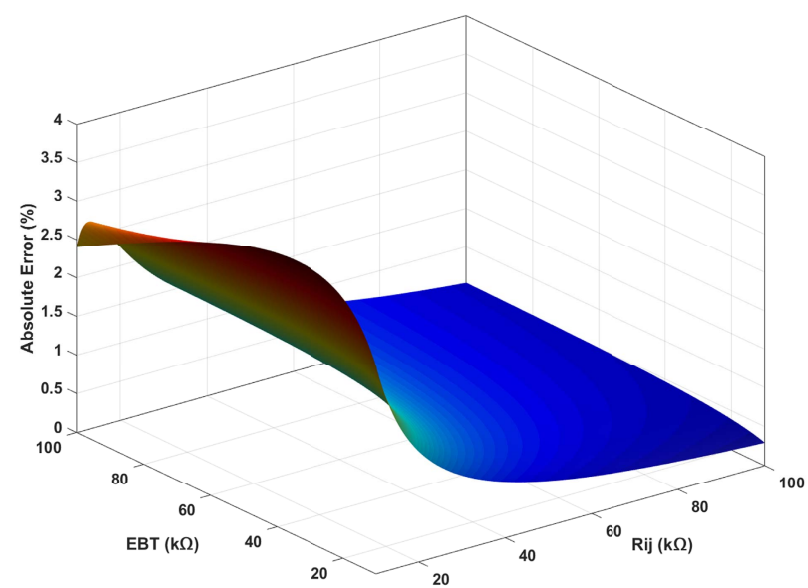

Fig. 9. Absolute error on readout of the EBT, with $\mathrm{M}=\mathrm{N}=60$. Minimal impedance $10 \mathrm{k} \Omega$.

and a large active area. Nevertheless, they have an intrinsic problem related to their resistive behavior and their interconnection circuit, which allows the occurrence of the crosstalk effect.

To develop a baropodometer based on a piezoresistive composite with an active area larger than $0.25 \mathrm{~m}^{2}$, a resistive array with almost 3600 sensors $(\mathrm{M}=\mathrm{N}=60)$ is required, as suggested by Giacomozzi et al. [22].

With this array size and according to the information contained in Fig. 7b, it is necessary to obtain a minimal impedance of $10 \mathrm{k} \Omega$. Then, using the Matlab model, with $R_{S W}=10 \Omega$, $R_{S}=10 \mathrm{k} \Omega$ and an input voltage of $V_{i}=5 \mathrm{~V}$ in a resistive array of $\mathrm{M}=\mathrm{N}=60$, we obtain a maximum absolute error of $3.56 \%$. The complete error behavior is shown in Fig. 9. Once again, the maximum percentage error differences of the Matlab and PSpice model errors with the operational amplifiers OPA37 and LM741 were $0.03 \%$ and $0.22 \%$, respectively. Furthermore, the model proposed by Wu et al. [14] with the same circuit parameters obtained a maximum error of 5.09\%, which is greater than the other simulated models.

\section{Conclusions}

In this study, we evaluated a number of circuits with respect to reducing the crosstalk effect in resistive sensor array. The circuit with the best response in terms of sensitivity and decreased crosstalk effect was the Improved Isolated Drive Feedback Circuit (IIDFC) proposed by Wu et al. [14]. Based on a nodal analysis, we developed a new mathematical model and, considering both EBT and non-scanned elements, determined the response as a function of array size, the conduction impedances of the switches, multiplexers and demultiplexers, and the resistive sensors themselves. The response of this new mathematical model to be close to that of the models simulated in the PSpice environment, with the maximum difference between the absolute mean errors being $0.03 \%$ with the opamp OPA37 and $0.16 \%$ with the opamp LM741. Hence, due to the low error between the mathematical and the PSpice models, the proposed model can be used as an accurate model for evaluating the $I I D F C$ performance. 
In the $I I D F C$, the resistive elements of the array must have a minimal allowable impedance to ensure a reduced crosstalk effect. The minimal impedance is directly related to the array size, whereby the larger the array size the greater the minimal allowable impedance in the array sensors. Many circuits have the same minimal impedance restriction, however, no authors have commented on this aspect of circuit characteristics.

When using the IIDFC and developing a piezoresistive composite with 3600 sensors $(M=N=60)$, this composite must have a minimal impedance of $10 \mathrm{k} \Omega$ to ensure a theoretical maximum absolute error of $3.56 \%$. Similarly, we can reduce the crosstalk effect using operational amplifiers with a high open loop gain, as well as switches, multiplexers and demultiplexers with conduction impedances less than $10 \Omega$. However, low impedances in these elements can lead the operational amplifier into saturation mode, thereby increasing the minimal allowable impedances allowed in the RSA.

\section{REFERENCES}

[1] H. Wang, J. Liu, and S. Chen, "An intelligent 3D force platform for plantar pressure distribution measurement," in Proc. Int. Conf. Mechatronics Autom. (ICMA), Changchun, China, Aug. 2009, pp. 4479-4483.

[2] N. Laowattanatham, K. Chitsakul, S. Tretriluxana, and C. Hansasuta, "Smart digital podoscope for foot deformity assessment," in Proc. 7th Biomed. Eng. Int. Conf. (BMEiCON), Fukuoka, Japan, Nov. 2014, pp. 1-5.

[3] D. Rosenbaum and H.-P. Becker, "Plantar pressure distribution measurements. Technical background and clinical applications," Foot Ankle Surgery, vol. 3, no. 1, pp. 1-14, 1997.

[4] A. H. A. Razak, A. Zayegh, R. K. Begg, and Y. Wahab, "Foot plantar pressure measurement system: A review," Sensors, vol. 12, no. 7, pp. 9884-9912, 2012

[5] F. Castro, "Aprimoramento de um baropodômetro eletrônico e análise de estabiliometria em voluntários com escoliose," M.S. thesis, Dept. Elect. Eng., Sao Paulo State Univ., São Paulo, Brazil, 2016. [Online]. Available: https://repositorio.unesp.br/handle/11449/138907

[6] M. F. R. Urban, "Implementação de um sistema eletrônico para avaliar a distribuição da força na região plantar de pacientes," Ph.D. dissertation, Dept. Electron., Sao Paulo State Univ., São Paulo, Brazil, 2015. [Online]. Available: https://repositorio.unesp.br/handle/11449/134100

[7] N. K. Rana, "Application of force sensing resistor (FSR) in design of pressure scanning system for plantar pressure measurement," in Proc. 2nd Int. Conf. Comput. Elect. Eng. (ICCEE), Dubai, United Arab Emirates, Dec. 2009, pp. 678-685.

[8] K. Petsarb, C. Apaiwong, C. Phairoh, R. Rattanakajornsak, Y. Kajornpredanon, and S. Daochai, "Low cost and customized plantar pressure analyzer for foot pressure image in rehabilitation foot clinic," in Proc. 5th Biomed. Eng. Int. Conf. (BMEiCON), Ubon Ratchathani, Thailand, Dec. 2012, pp. 1-4.

[9] S. Khan, L. Lorenzelli, and R. S. Dahiya, "Bendable piezoresistive sensors by screen printing MWCNT/PDMS composites on flexible substrates," in Proc. 10th Conf. Ph.D. Res. Microelectron. Electron. (PRIME), Grenoble, France, Jun./Jul. 2014, pp. 1-4.

[10] C.-W. Ma, C.-M. Chang, T.-H. Lin, and Y.-J. J. Yang, "Highly sensitive tactile sensing array realized using a novel fabrication process with membrane filters," J. Microelectromech. Syst., vol. 24, no. 6, pp. 2062-2070, Dec. 2015.

[11] S. Stassi et al., "Wearable and flexible pedobarographic insole for continuous pressure monitoring," in Proc. IEEE SENSORS, Baltimore, MD, USA, Nov. 2013, pp. 1-4.

[12] H. Liu, Y.-F. Zhang, Y.-W. Liu, and M.-H. Jin, "Measurement errors in the scanning of resistive sensor arrays," Sens. Actuators A, Phys., vol. 163, no. 1, pp. 198-204, Sep. 2010.

[13] R. S. Saxena, N. K. Saini, and R. K. Bhan, "Analysis of crosstalk in networked arrays of resistive sensors," IEEE Sensors J., vol. 11, no. 4, pp. 920-924, Apr. 2011.

[14] J. Wu, L. Wang, and J. Li, "Design and crosstalk error analysis of the circuit for the 2-D networked resistive sensor array," IEEE Sensors J., vol. 15, no. 2, pp. 1020-1026, Feb. 2015.
[15] J.-S. Kim, D.-Y. Kwon, and B.-D. Choi, "High-accuracy, compact scanning method and circuit for resistive sensor arrays," Sensors, vol. 16, no. 2, p. $155,2016$.

[16] J.-F. Wu, "Scanning approaches of 2-D resistive sensor arrays: A review," IEEE Sensors J., vol. 17, no. 4, pp. 914-925, Feb. 2017.

[17] T. D'Alessio, "Measurement errors in the scanning of piezoresistive sensors arrays," Sens. Actuators A, Phys., vol. 72, no. 1, pp. 71-76, Jan. 1999.

[18] R. S. Saxena, R. K. Bhan, and A. Aggrawal, "A new discrete circuit for readout of resistive sensor arrays," Sens. Actuators A, Phys., vol. 149, no. 1, pp. 93-99, Jan. 2009.

[19] J. Wu, L. Wang, and J. Li, "VF-NSE method measurement error analysis of networked resistive sensor array," Sens. Actuators A, Phys., vol. 211, pp. 45-50, May 2014.

[20] J. Wu, L. Wang, J. Li, and A. Song, "A novel crosstalk suppression method of the 2-D networked resistive sensor array," Sensors, vol. 14 no. 7, pp. 12816-12827, 2014.

[21] J.-F. Wu, F. Wang, Q. Wang, J.-Q. Li, and A.-G. Song, "An improved zero potential circuit for readout of a two-dimensional resistive sensor array," Sensors, vol. 16, no. 12, p. 2070, 2016.

[22] C. Giacomozzi, N. Keijsers, T. Pataky, and D. Rosenbaum, "International scientific consensus on medical plantar pressure measurement devices: Technical requirements and performance," Ann. Istituto Superiore Sanità, vol. 48, no. 3, pp. 259-271, Jan. 2012.

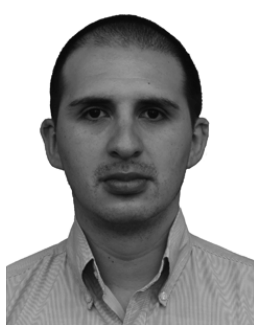

Fabian Castro received the B.S. degree in electronic engineering from the Pedagogical and Technological University of Colombia, Sogamoso, Colombia, in 2011, and the M.S. degree in electrical engineering from São Paulo State University, Ilha Solteira, Brazil, in 2016, where he is currently pursuing the $\mathrm{Ph} . \mathrm{D}$. degree in electrical engineering. His main research interests include piezoresistive composites, sensor networks, biomedical devices, and artificial neural networks.

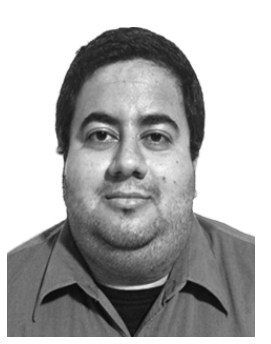

Thiago Pentiado received the B.S. degree in electrical engineering from São Paulo State University, Ilha Solteira, Brazil, in 2015, where he is currently pursuing the M.S. degree in electrical engineering. His main research interests include biomedical devices, digital and analogue instrumentation, sensor networks, and digital signal processing.

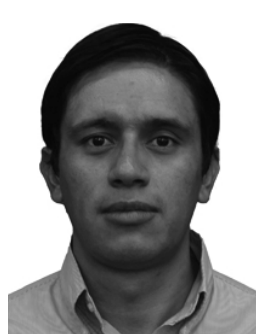

Jorge Blanco received the B.S. degree in electronic engineering from the Pedagogical and Technological University of Colombia, Sogamoso, Colombia, in 2012, and the M.S. degree in electrical engineering from São Paulo State University, Ilha Solteira, Brazil, in 2016, where he is currently pursuing the $\mathrm{Ph} . \mathrm{D}$. degree in electrical engineering. His main research interests include SSVEP-BCI, functional electrical stimulation, biomedical devices, and artificial neural networks.

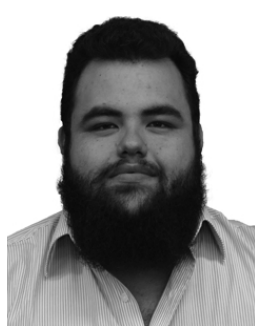

Ricardo Xavier received the B.S. degree in mathematics from the Fernandópolis Educational Foundation, Fernandópolis, Brazil, in 2014, and the M.S. degree in electrical engineering from São Paulo State University, Ilha Solteira, Brazil, in 2016, where he is currently pursuing the Ph.D. degree in electrical engineering. His main research interests include biomechatronics, upper limb prosthesis, electromyography, biomedical devices, and artificial neural networks. 


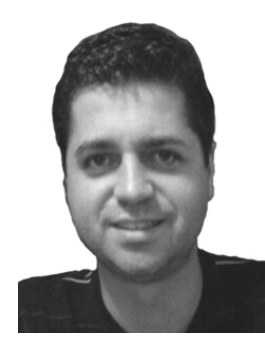

Marcelo Sanches received the B.S. degree in electrical engineering from the University Center of the Educational Foundation of Barretos in 2003, and the M.S. and Ph.D. degrees in control and automation in biomedical engineering from São Paulo State University (UNESP), Ilha Solteira, Brazil, in 2007 and 2012, respectively. He did a Ph.D. internship at the Bioengineering Centre, Fnd. Don Carlo Gnocchi IRCCS ONLUS, Milan, Italy, in the Fondazione Don Gnocchi (2011). He started his career as a Professor with the Department of Electrical Engineering, UNESP, in 2012. He has been a Professor and a Researcher with the Laboratory of Instrumentation and Biomedical Engineering. His research interests include biomedical instrumentation, and control and rehabilitation engineering.

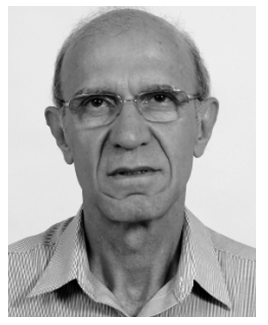

Aparecido de Carvalho received the B.S. degree in electrical engineering from the University of São Paulo in 1976, the M.S. degree in biomedical engineering from the Federal University of Rio de Janeiro in 1979, and the Ph.D. degree in physics from the University of São Paulo in 1987. He started his career as an Assistant Professor with the Department of Electrical Engineering, São Paulo State University, Brazil, in 1980. From 1993 to 1994, he was a Post-Doctoral Scientist with the University of Wisconsin-Madison, investigating a method for measuring of X-ray intensity in the medical diagnostic range by pyroelectric sensors and a method for extending the practical range of conductive polymer sensors for measuring contact force. Since 2005, he has been a Professor. His research interests include biomedical instrumentation, rehabilitation engineering, and functional electrical stimulation. He was a member of the Advisory Committee of the National Council for Scientific and Technological Development-CNPq, Brazil, in the area of electrical and biomedical engineering from 2008 to 2010. He was the Secretary of the Brazilian Society of Biomedical Engineering in the 2011-2012 biennium. 